Supporting Information for the article:

\title{
Breath Analysis Based on Surface Enhanced Raman Scattering Sensors Distinguishes Early and Advanced Gastric Cancer Patients From Healthy Persons
}

Yunsheng Chen ${ }^{1,2}$, Yixia Zhang ${ }^{1}$, Fei Pan ${ }^{1}$, Jie Liu ${ }^{3}$, Kan Wang ${ }^{1}$, Chunlei Zhang ${ }^{1}$, Shangli Cheng ${ }^{1}$, Lungen $\mathrm{Lu}^{4}$, Wei Zhang ${ }^{5}$, Zheng Zhang ${ }^{6}$, Xiao Zhi ${ }^{1}$, Qian Zhang ${ }^{1}$, Gabriel Alfranca ${ }^{1,7}$, Jesús M. de la Fuente ${ }^{7}$, Di Chen ${ }^{1}$ and Daxiang Cui ${ }^{1,2,8}$

${ }^{1}$ Institute of Nano Biomedicine and Engineering, Shanghai Engineering Research Center for Intelligent Instrument for Diagnosis and Therapy, Key Laboratory for Thin Film and Microfabrication of Ministry of Education, Department of Instrument Science and Engineering, School of Electronic Information and Electronical Engineering, 800 Dongchuan Road, Shanghai Jiao Tong University, Shanghai 200240, P. R. China

${ }^{2}$ School of Biomedicine Engineering, Shanghai Jiao Tong University, Shanghai 200240, P. R. China

${ }^{3}$ Department of Digestive Diseases, Huashan Hospital, Fudan University, Shanghai 210040, P. R. China

${ }^{4}$ Department of Gastroenterology, Shanghai First People's Hospital, Shanghai Jiao Tong University School of Medicine, Shanghai 200080, P. R. China

${ }^{5}$ Department of Orthopedics, Shanghai Sixth People's Hospital, Shanghai Jiao Tong University, School of Medicine Shanghai 200233, P. R. China

${ }^{6}$ Department of Plastic and Reconstructive Surgery, Shanghai Ninth People's Hospital, Shanghai Jiao Tong University School of Medicine, Shanghai 200011, P. R. China

${ }^{7}$ Instituto de Ciencia de Materiales de Aragón (ICMA-CSIC), Universidad de Zaragoza, 50009-Zaragoza, Spain

${ }^{8}$ National Center for Translational Medicine, Shanghai Jiao Tong University, Shanghai 200240, P. R. China

* Corresponding author. Tel: 0086-21-34206886. Fax: 0086-21-34206886. E-mail: dxcui@sjtu.edu.cn 


\section{S1. Figure captions}

Figure S1 A schematic illustration of SERS "hotspots" on the SERS sensor.

Figure S2 Schematic diagrams of $N_{\text {SERS }}(\mathrm{A})$ and $N_{b l u k}(\mathrm{~B})$.

Figure S3 SERS spectra of $\left(10^{-5} \mathrm{M}\right)$ naphthalene on SERS sensor (black curve) and Raman spectra of bulk naphthalene on au film substrate (red curve)

Figure S4 (A) The photo of the SERS active area in SERS sensor; (B) All SERS spectra of R6G obtained during Raman mapping.

Figure S5 (A) The original Raman spectra of the mixture of biomarker standards corresponding to the healthy people (a), the patients with EGC (b) and AGC (c), and a control sample (d). (B) Raman spectra of acetone (1), pivalic acid (2), hexanol (3), menthol (4) and alcohol (5) in glass capillary.

Figure S6 the Raman spectra of VOC biomarker standards (red curve) and the SERS spectra of gasous VOC biomarker standards (black curve).

Figure S7 The SERS spectra from the two-day analyses of a same breath sample (A), and the two-point analyses of a same sensor (B). The breath samples were from patients with EGC and controls, respectively. 


\section{S2 The details of processing Raman spectra}

14 VOC biomarkers have excellent solubility in organic solvents such as ethanol, chloroform. However, most organic solvents have toxicity or too many Raman characteristic peaks. Among various organic solvents, ethanol is considered as the best option because it is almost non-toxic and few effects of Raman detection. After a polynomial baseline correction, the obtained Raman spectra of the mixture of biomarker standards had the similar peaks to one of the control (shown in Figure $\mathrm{S} 1 \mathrm{~A})$. This phenomenon is attributed to that ethanol as the solvent was the main component $(99 \%, \mathrm{v} / \mathrm{v})$. Therefore, the Raman spectra should be subtracted the background before used to diagnosis. It is important to mention that the intensity of an analyte band is linearly proportional to the amount of the analyte. In another word, ethanol contributed the almost same Raman signal (both intensity and Raman shift) in all Raman spectra. Additionally, a peak appears in the Raman spectrum of ethanol at $884 \mathrm{~cm}^{-1}$, which is due to the symmetric CCO stretch. ${ }^{1}$ Compared to the oxygen-containing VOC biomarkers, ethanol has a signature peak at $884 \mathrm{~cm}^{-1}$ (shown in Figure S1B). Therefore, ethanol can act as an internal standard to correct the Raman spectra and provide a more robust quantification method by subtracting the spectrum of control. $^{2}$

\section{S3. Experimental details:}

\section{S3.1 Synthesis of Graphene Oxide (GO):}

GO was prepared from natural graphite powder via acid-oxidation according to a modified Hummers method as mentioned in our previous papers. ${ }^{3-6}$ Briefly, $2 \mathrm{~g}$ graphite and $1 \mathrm{~g} \mathrm{NaNO}_{3}$ were put into a flask cooled at $0{ }^{\circ} \mathrm{C}$. Then, $50 \mathrm{~mL}$ concentrated $\mathrm{H}_{2} \mathrm{SO}_{4}$ was carefully added to the mixture. The mixture was stirred for 30 min at $5{ }^{\circ} \mathrm{C}$. Subsequently, $7 \mathrm{~g}_{\mathrm{KMnO}_{4}}$ was added to the reaction system 
step-wisely over $1 \mathrm{~h}$ with temperature below $20{ }^{\circ} \mathrm{C}$. The temperature of the reaction system was then raised to $35{ }^{\circ} \mathrm{C}$ and stirred for $2 \mathrm{~h}$. Afterwards, $90 \mathrm{~mL} \mathrm{H} \mathrm{H}_{2} \mathrm{O}$ was slowly added into the paste like product, and the temperature of the reaction system jumped to $70{ }^{\circ} \mathrm{C}$ instantly. Finally, $7 \mathrm{~mL} 30 \% \mathrm{H}_{2} \mathrm{O}_{2}$ and $55 \mathrm{~mL} \mathrm{H} \mathrm{H}_{2} \mathrm{O}$ were poured into the reaction system resulting in the formation of bright yellow suspension. The solid product, graphite oxide, was separated from the reaction mixture by filtration. The yellow-brown solid powders were washed for three times with diluted $\mathrm{HCl}(3 \%)$, and then dispersed in $\mathrm{H}_{2} \mathrm{O}$. Exfoliation of graphite oxide was approached by sonicating the graphite oxide in water at room temperature for $1 \mathrm{~h}$ generating homogeneous graphene oxide dispersions.

\section{S3.2 Purification of GO:}

GO homogeneous solution was washed 3 times by diluted different concentrations of $\mathrm{HCl}(5 \%)$ and separated by a high-speed centrifugation (8000 rpm, $20 \mathrm{~min})$. Then, GO was repeatedly re-dispersed in water and separated by high-speed centrifugation (10000 rpm, $30 \mathrm{~min}$ ), until most of GO could not been separated. Subsequently, GO was further purified by dialysis in water for one week.

\section{S4 Calculation of $N_{S E R S}$ and $N_{b u l k}$}

The calculation of enhancement factor (EE) was according to same procedures based on the published literatures. ${ }^{7-9}$ At first, the area of the laser spot size $\left(S_{\text {laser }}\right)$ was ca. $1 \mu \mathrm{m}^{2}$ using the $100 \times$ objective lens in this work. As shown in Figure S2A, $1 \mu \mathrm{L}$ of R6G solution $\left(10^{-6} \mathrm{M}\right)$ uniformly spread and dried in surface of prepared SERS sensor with a single-molecule layer. According a lot of reported works, the area of single R6G molecule $\left(S_{R 6 G}\right)$ was $10^{4} \mathrm{~nm}^{2}$. Therefore, according the Equation (1), $N_{\text {SERS }}$ was $10^{2}$. Shown in Figure S1B, the bulk R6G molecules were disorder using R6G powder. Obviously, the Equation (1) was not suit for calculating $N_{\text {bulk }}$. Generally, the 
molecules on the top layer could be excited by laser, and these molecules could be countered into $N_{b u l k}$. Equation (2) was used to calculate the $N_{b u l k}$, where A was Avogadro constant $\left(6 \times 10^{23}\right), h$ was the laser penetration depth $(2 \mu \mathrm{m}), \mathrm{S}$ was the area of laser spot (approximately $\left.1 \mu \mathrm{m}^{2}\right), \rho_{\mathrm{R} 6 \mathrm{G}}$ was the density of R6G $\left(0.79 \mathrm{~g} / \mathrm{cm}^{3}\right), \mathrm{M}_{\mathrm{R} 6 \mathrm{G}}$ was molar mass of R6G (479 g/mol). Therefore, $N_{\text {bulk }}$ was $5 \times 10^{9}$.

$$
\begin{aligned}
& N_{\text {SERS }}=S_{\text {laser }} / \mathrm{S}_{\mathrm{R} 6 \mathrm{G}} \\
& N_{\text {bulk }}=\mathrm{A} \times\left(\mathrm{S} \times h \times \rho_{\mathrm{R} 6 \mathrm{G}}\right) / \mathrm{M}_{\mathrm{R} 6 \mathrm{G}}
\end{aligned}
$$

\section{S5 Calculation of EF based on naphthalene (NT)}

The $10^{-5} \mathrm{M}$ NT solution was introduced to the EF calculation. $1 \mu \mathrm{L}$ NT solution was dropped on the SERS sensor and drying at room temperature. As shown in Figure S3, four characteristics bands of naphthalene were observed in both SERS and Raman spectra. ${ }^{10}$ the band at 1578,1380 and $1018 \mathrm{~cm}^{-1}$ were attributed to the ring skeletal vibration of naphthalene, the band at $760 \mathrm{~cm}^{-1}$ was assigned to $\mathrm{CH}$ bending. ${ }^{11}$ The $\mathrm{EF}$ calculation was also acroding to the relationship.

$$
\mathrm{EF}^{\prime}=\left(\dot{I}_{\text {SERS }} / \dot{I}_{\text {bulk }}\right)\left(N_{\text {bulk }} / N_{\text {SERS }}\right)
$$

Where, $I_{\text {SERS }}$ was the intensity of the band at $1380 \mathrm{~cm}^{-1}$ in SERS spectrum (black curve in Figure S3), while $\dot{I}_{\text {bulk }}$ represented the intensity of same band in Raman spectrum (red curve in Figure S3). $N_{\text {SERS }}$ was the number of NT molecule contributing to the SERS signal under laser beam and $N_{b u l k}$ was the number of solid NT molecules contributing to the Raman signal. $N_{\text {SERS }}$ and $N_{\text {bulk }}$ could be calculated as same as S4.

$$
\begin{aligned}
& N_{\text {SERS }}^{\prime}=S_{\text {laser }} / \mathrm{S}_{\mathrm{NT}} \\
& N_{\text {bulk }}^{\prime}=\mathrm{A} \times\left(\mathrm{S}_{\text {laser }} \times h \times \rho_{\mathrm{NT}}\right) / \mathrm{M}_{\mathrm{NT}}
\end{aligned}
$$

Where, $\mathrm{S}_{\mathrm{NT}}$ was the area of a naphthalene molecule (approximately $1 \mathrm{~nm}^{2}$ ) $;^{12} h$ was the laser penetration depth $(2 \mu \mathrm{m}) ; \rho_{\mathrm{NT}}$ is the density of NT molecules $(1.162$ $\left.\mathrm{g} / \mathrm{cm}^{3}\right)$ and $\mathrm{M}_{\mathrm{NT}}$ was molar mass of R6G (128 $\left.\mathrm{g} / \mathrm{mol}\right)$. Therefore, $N_{\text {SERS }}$ was $10^{6}$ and $N_{\text {bulk }}$ was $2 \times 10^{10}$. Therefore, EF' was $1.02 \times 10^{6}$. 


\section{S6 The preparation of TEM sample}

(1) $1 \mu \mathrm{L}$ GO $(0.02 \mathrm{mg} / \mathrm{ml})$ was dropped on a lacey support film and drying at room temperature. (2) the lacey support film were placed in a cleaned glass Petri dish inside a larger glass beaker containing hydrazine monohydrate $(100 \mu \mathrm{L}$, wt. $\%=85 \%)$ at $40{ }^{\circ} \mathrm{C}$ for $2 \mathrm{~h}$. (3) $1 \mu \mathrm{L} \mathrm{HAuCl}_{4}(5 \mathrm{mM})$ was cover the RGO (RGO display light black color after reduction). After $1 \mathrm{~min}$, the $\mathrm{HAuCl}_{4}$ solution was carefully removed by a filter paper. The TEM samples were prepared after they dried at a $\mathrm{N}_{2}$ environment. 


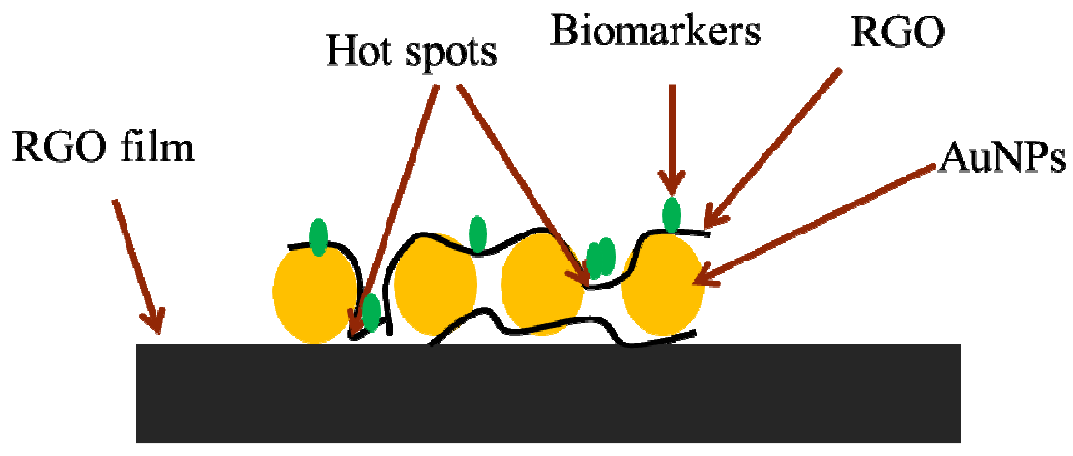

Figure S1 


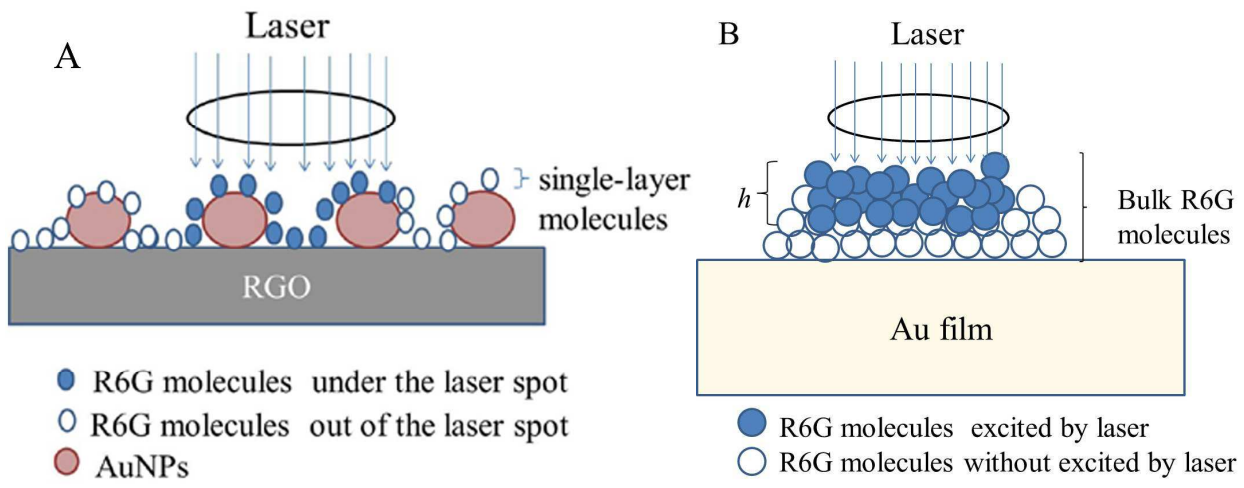

Figure S2 


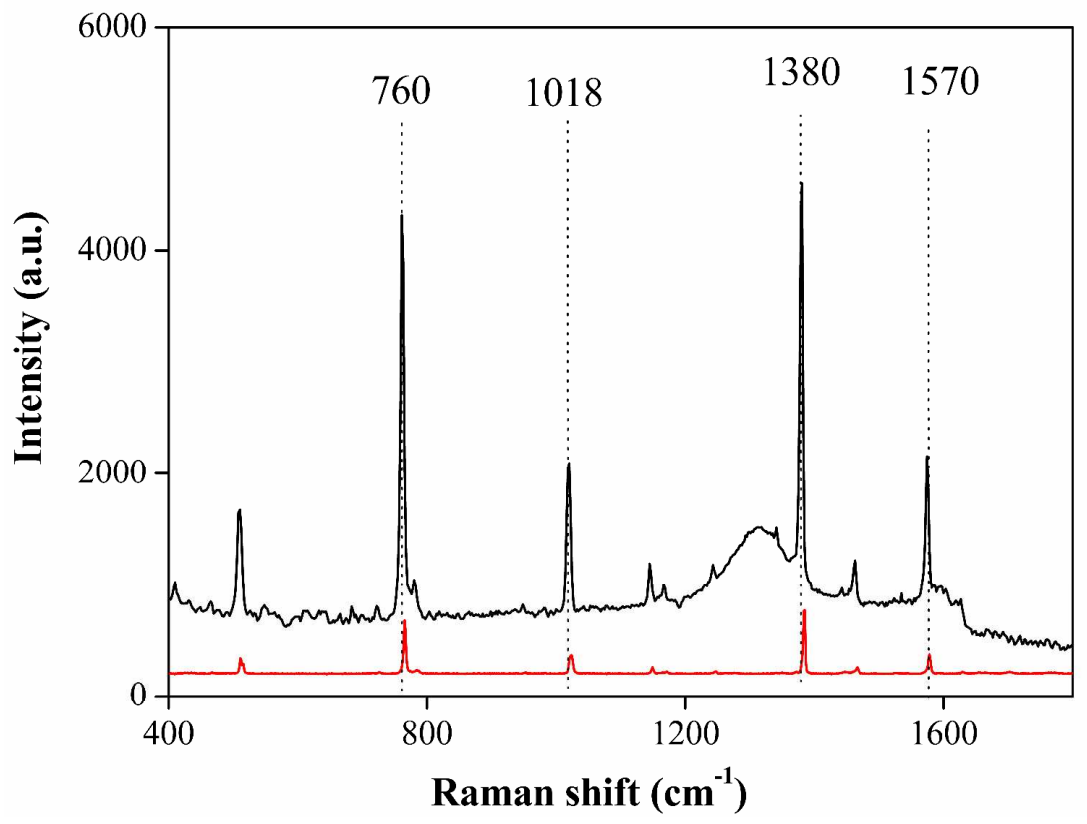

Figure S3 

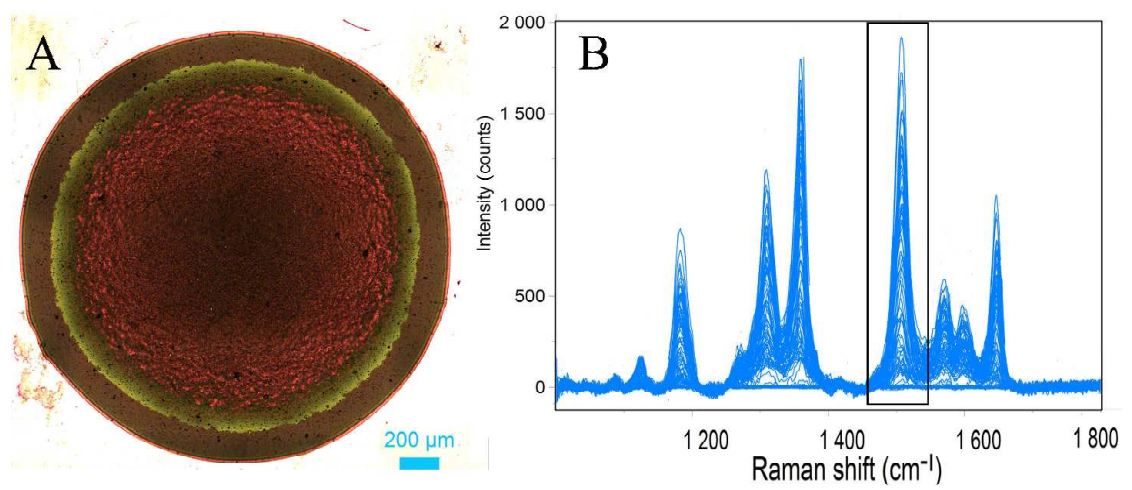

Figure S4 

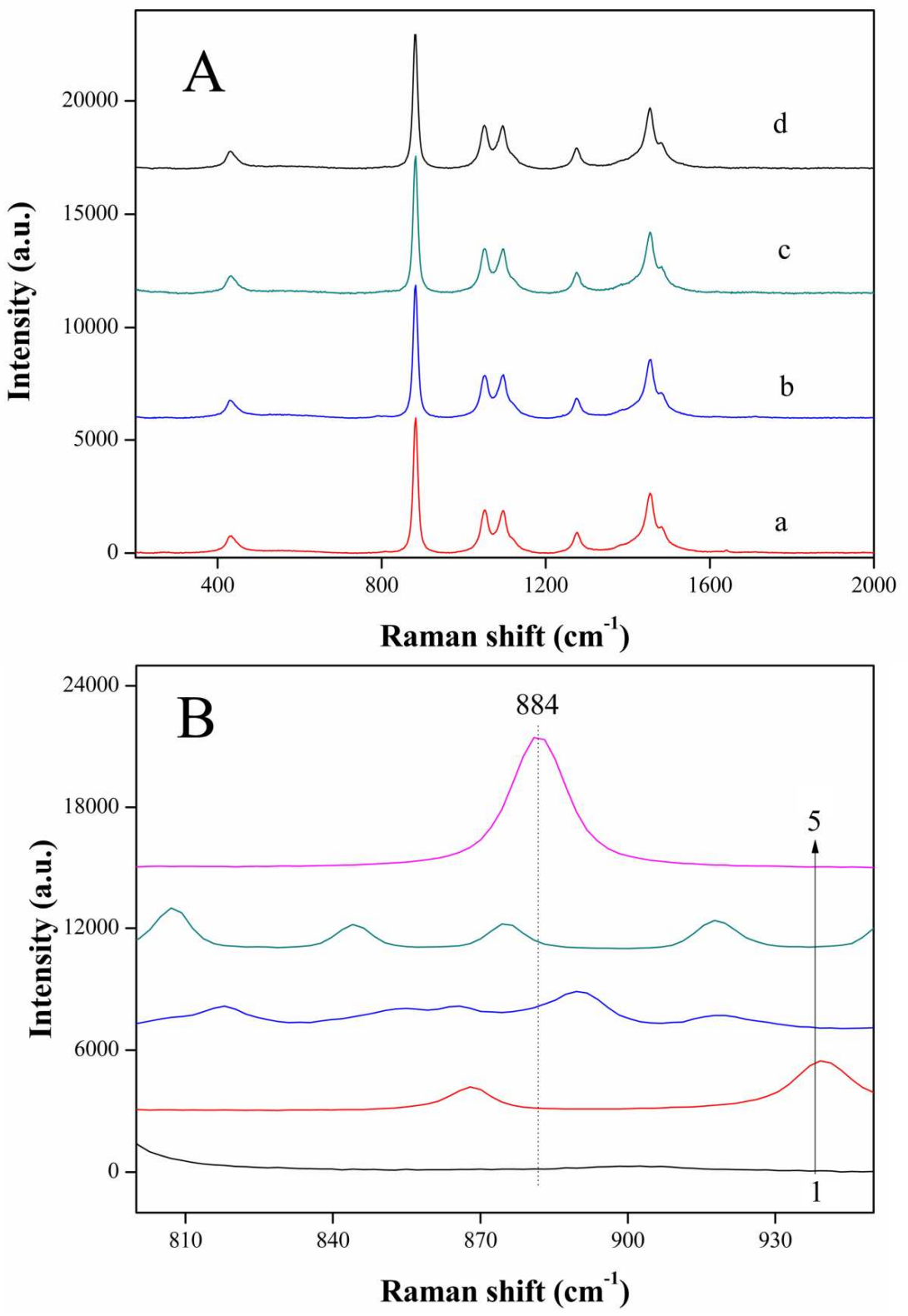

Figure S5 

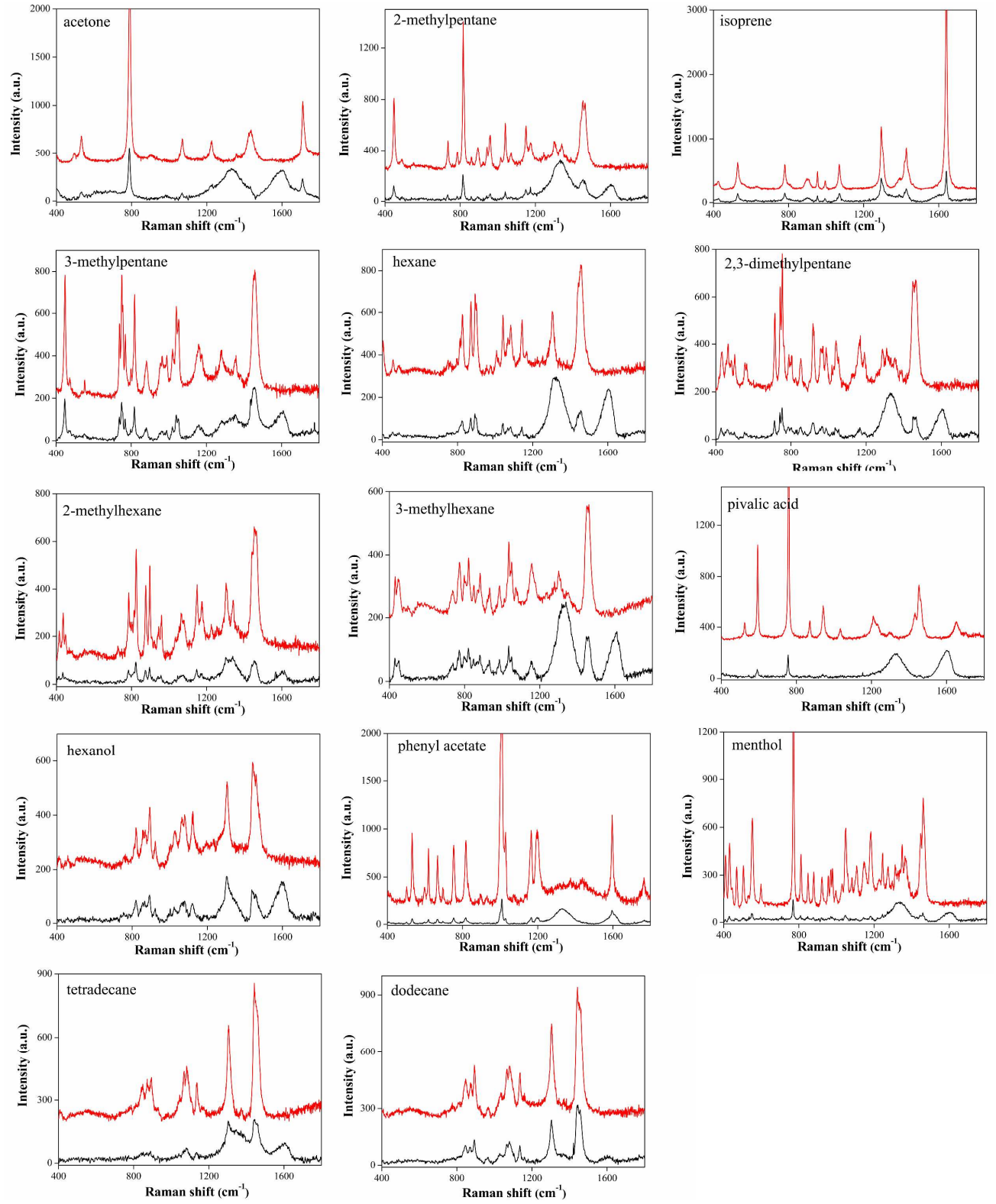

Figure S6 

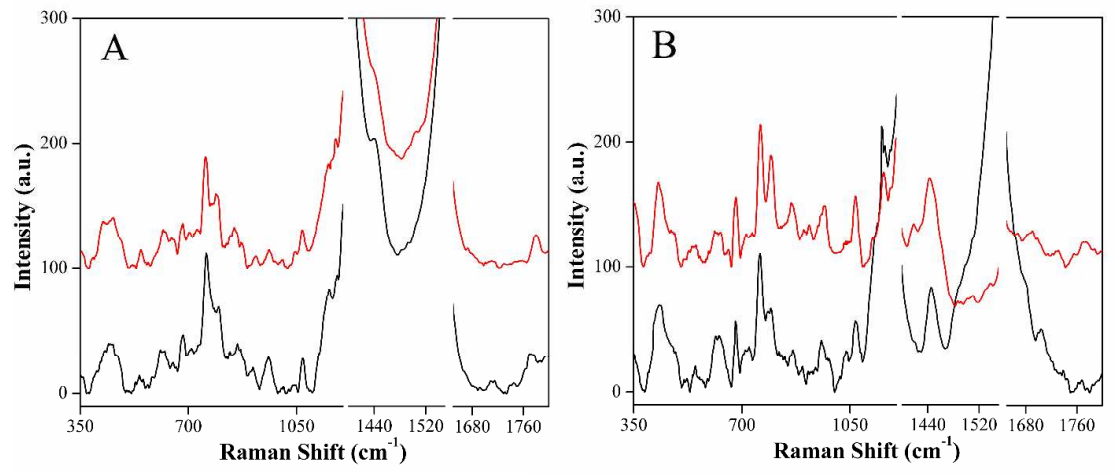

Figure S7 
Table S1

\begin{tabular}{cccccc}
\hline Peak & Retention & Compound & \multicolumn{3}{c}{ Relative concentration $^{\mathrm{a}}$} \\
\cline { 3 - 5 } & time (min) & & Healthy & Early & Advanced \\
\hline 1 & 4.89 & Acetone & $0.174 \pm 0.0885$ & $9.7 \pm 1.9$ & $0.160 \pm 0.0313$ \\
2 & 7.94 & 2-Methylpentane & $0.142 \pm 0.0391$ & $0.179 \pm 0.140$ & $0.210 \pm 0.623$ \\
3 & 8.02 & Isoprene & $2.32 \pm 0.1490$ & $0.0522 \pm 0.0396$ & $0 \pm 0$ \\
4 & 8.39 & 3-Methylpentane & $0.0579 \pm 0.0819$ & $0.0443 \pm 0.0294$ & $1.91 \pm 0.563$ \\
5 & 8.86 & 1-Hexane & $0.0255 \pm 0.361$ & $0.0947 \pm 0.0775$ & $6.49 \pm 0.276$ \\
6 & 9.88 & 2,3-Dimethylpentane & $0 \pm 0$ & $0.101 \pm 0.116$ & $0.499 \pm 0.0226$ \\
7 & 11.13 & 2- Methylhexane & $0.438 \pm 0.391$ & $0.375 \pm 0.318$ & $1.59 \pm 0.0613$ \\
8 & 11.40 & 3- Methylhexane & $0 \pm 0$ & $0.0185 \pm 0.0212$ & $0.888 \pm 0.213$ \\
9 & 19.46 & Pivalic acid & $0.241 \pm 0.110$ & $0.547 \pm 0.500$ & $0.112 \pm 0.000$ \\
10 & 20.13 & n-Hexanol & $0.956 \pm 0.041$ & $1.011 \pm 0.134$ & $1.000 \pm 0.071$ \\
11 & 22.61 & Phenyl acetate & $0.190 \pm 0.0333$ & $1.15 \pm 1.18$ & $0.186 \pm 0.0129$ \\
12 & 23.27 & Menthol & $2.31 \pm 2.64$ & $0.452 \pm 0.440$ & $0.222 \pm 0.00400$ \\
13 & 25.64 & n-Tetradecane & $0.292 \pm 0.0471$ & $7.98 \pm 0.313$ & $1.21 \pm 0.0151$ \\
14 & 25.81 & Dodecane & $0.0724 \pm 0.102$ & $0.212 \pm 0.159$ & $0.233 \pm 0.00156$ \\
\hline & 0 & & & &
\end{tabular}

${ }^{\text {a }}$ calculated as the ratio of peak areas of each compound to the one of n-Hexanol. 


\section{References}

1. Dollish, F. R.; Fateley, W. G.; Bentley, F. F. Characteristic Raman Frequencies of Organic Compounds. Wiley: 1974.

2. Beier, B. D.; Berger, A. J. Method for Automated Background Subtraction From Raman Spectra Containing Known Contaminants. Analyst 2009, 134, 1198-1202.

3. Hummers Jr, W. S.; Offeman, R. E. Preparation of Graphitic Oxide. J. Am. Chem. Soc. 1958, 80, 1339-1339.

4. Zhang, J.; Yang, H.; Shen, G.; Cheng, P.; Zhang, J.; Guo, S. Reduction of Graphene Oxide via L-Ascorbic Acid. Chem. Commun. 2010, 46, 1112-1114.

5. Zhang, J.; Shen, G.; Wang, W.; Zhou, X.; Guo, S. Individual Nanocomposite Sheets of Chemically Reduced Graphene Oxide and Poly (N-Vinyl Pyrrolidone): Preparation and Humidity Sensing Characteristics. J. Mater. Chem. 2010, 20, 10824-10828.

6. Wang, X.; Wang, Q. X.; Wang, Q. H.; Gao, F.; Gao, F.; Yang, Y. Z.; Guo, H. X. Highly Dispersible and Stable Copper Terephthalate Metal-Organic Framework-Graphene Oxide Nanocomposite for An Electrochemical Sensing Application. Acs. Appl. Mater. Inter. 2014, 6, 11573-11580.

7. Guo, B.; Han, G.; Li, M.; Zhao, S. Deposition of The Fractal-Like Gold Particles onto Electrospun Polymethylmethacrylate Fibrous Mats and Their Application in Surface-Enhanced Raman Scattering. Thin Solid Films 2010, 518, 3228-3233.

8. Yi, Z.; Chen, S. J.; Chen, Y.; Luo, J. S.; Wu, W. D.; Yi, Y. G.; Tang, Y. J. Preparation of Dendritic $\mathrm{Ag} / \mathrm{Au}$ Bimetallic Nanostructures and Their Application in Surface-Enhanced Raman Scattering. Thin Solid Films 2012, 520, 2701-2707.

9. Xiao, G. N.; Man, S. Q. Surface-Enhanced Raman Scattering of Methylene Blue Adsorbed on Cap-Shaped Silver Nanoparticles. Chem. Phys. Lett. 2007, 447, 305-309.

10. Huang, Z.; Qu, L.; Shi, G.; Chen, F. e.; Hong, X. Electrochemical Polymerization of Naphthalene in The Electrolyte of Boron Trifluoride Diethyl Etherate Containing Trifluoroacetic Acid and Polyethylene Glycol Oligomer. J. Electroanal. Chem. 2003, 556, 159-165.

11. Selvakumar, S.; Sivaji, K.; Arulchakkaravarthi, A.; Balamurugan, N.; Sankar, S.; Ramasamy, P. Growth of High-Quality Naphthalene Single Crystals Using Selective Self-Seeding Vertical Bridgman Technique (SSVBT) and Its Characterization. J. Cryst. Growth 2005, 282, 370-375.

12. Oliferova, L.; Statkus, M.; Thysin, G.; Zolotov, Y. A. In New Sorbents for Preconcentration of Hydrophobic Organic Compounds in Flow Analysis Systems. Dokl. Chem. 2005,401, 62-65. 